\title{
PARTICIPACIÓN DE ASESORES INDÍGENAS EN UNA UNIVERSIDAD CONVENCIONAL. EXPERIENCIAS, SUJETOS Y SABERES
}

\section{INDIGENOUS PARTICIPATION IN A CONVENTIONAL UNIVERSITY. EXPERIENCES, SUBJECTS AND KNOWLEDGE}

\author{
Laura Liliana Rosso ${ }^{1}$
}

Fecha de recepción: 24-06-2016

Fecha de aceptación y versión final: 30-08-2016

Resumen: En este artículo propongo presentar avances preliminares de un estudio sobre participación indígena en una universidad convencional, la Universidad Nacional del Nordeste (Argentina), en la que se implementa un programa institucional que tiene entre sus objetivos la inclusión de indígenas a carreras de grado, así como su participación en la gestión del mismo. Participación que se promueve buscando aportar a la concreción del derecho definido en normativas internacionales, nacionales, provinciales; y en una lectura de los recorridos alcanzados por las organizaciones indígenas de la provincia del Chaco.

Se focalizará aquí en la descripción de espacios y experiencias de participación surgidas en el marco del "Programa Pueblos Indígenas" de la Universidad referida. Los sujetos son representantes de organizaciones indígenas que integran la comisión asesora del programa, quienes ocupan esa posición en virtud de sus trayectorias en organizaciones socio étnicas, son adultos y no pertenecen a ningún claustro universitario. Interesa iniciar una indagación sobre los saberes vinculados a la organización, la lucha y la política que estos sujetos poseen, así como las habilidades que ponen en práctica en las instancias de gestión de las que son parte.

Los tópicos planteados permiten presentar, al final, reflexiones acerca de las posibilidades de diálogos entre culturas, y el lugar de los "informantes" e investigadora-actora en este estudio.

Palabras clave: universidad - participación indígena - saberes socialmente productivos

Abstract: On this article I suggest to present preliminary progress in a study about Indigenous participation in a conventional university, the Universidad Nacional del Nordeste (Argentina), where an institutional program which objectives, among others, have to do with indigenous inclusion in undergraduate careers is implemented, as well as their participation in its management.

This participation is promoted seeking to contribute to the concretion of the right defined in international, national and provincial state regulations; and on a reading of the journeys accomplished by the indigenous organizations in the Chaco province.

Descriptions of spaces and participation experiences framed the 'Indigenous Peoples Program' on the above-mentioned University. The subjects are indigenous representatives who are members of the advisory commission of the program. They hold that position due to their trajectory in socio-ethnic organizations, they are adults but they don't belong to any university faculty. It is interesting to initiate an inquiring over the knowledge related to the organization, the struggle and the policy that these individuals bare, as well as the abilities they set on over the management instances they are part of.

\footnotetext{
${ }^{1}$ Licenciada en Ciencias de la Educación, Magíster en Antropología Social. Investigadora del IIE, Profesora Adjunta de la Licenciatura en Ciencias de la Educación, Facultad de Humanidades, Universidad Nacional del Nordeste. Pertenencia institucional: Instituto de Investigaciones en Educación (IIE). Facultad de Humanidades. Universidad Nacional del Nordeste. Correo electrónico: lauralirosso@gmail.com
} 
At the end the posed topics allow to present reflections over the possibilities of dialogues between cultures and the place of the 'informants' and the researcher-performer on this study.

Key words: university - indigenous participation - socially productive knowledge

\section{Introducción}

Una política inclusiva de indígenas en la universidad puede resultar a los ojos de la opinión pública un acto de reparación histórica en una provincia argentina como el Chaco, donde la legislación que reconoce derechos a este colectivo es avanzada en sus enunciados. Sin embargo, habilitar su participación en distintos ámbitos de la universidad, junto con reconocer a sus representantes como interlocutores válidos en ese proceso, son cuestiones de difícil comprensión tanto para la opinión pública como para los distintos actores que componen la universidad.

Este artículo se propone presentar avances preliminares de un estudio sobre participación indígena en una universidad convencionali', la Universidad Nacional del Nordeste, ubicada en las provincias de Chaco y Corrientes (Argentina). Institución en la que se implementa el Programa Pueblos Indígenas (en adelante PPI) que tiene entre sus objetivos la inclusión de indígenas a carreras de grado así como su participación en la gestión del mismo. Esta última se promueve buscando aportar a la concreción del derecho definido en normativas internacionales, nacionales y provinciales, al tiempo que se basa en una lectura de los recorridos alcanzados por las organizaciones indígenas de la provincia del Chaco (Resol. No. 733/10 C.S.).

Se analizará aquí la experiencia de funcionamiento de la comisión asesora del programa mencionado, comisión compuesta por cinco representantes de los pueblos indígenas del Chaco - tres qom, un moqoit y un wichí - ; quienes ocupan esta posición en virtud de sus trayectorias en organizaciones étnicas. Además de este antecedente son trabajadores de áreas de educación y/o cultura del Estado provincial; profesan el culto evangélico ${ }^{i i}$ y no pertenecen a ningún claustro universitario. Se sostiene aquí que dichos componentes de sus trayectorias coadyuvan en su formación como representantes indígenas, esto porque se adopta el concepto de formación de Ferry (2008:54) según el cual las personas se forman por mediación. Las mediaciones son diversas, exceden a la enseñanza y el aprendizaje escolar. Desde este enfoque se valora no sólo a profesores y textos como mediadores de formación, sino también a las circunstancias, los accidentes de la vida, la relación con otros, en la vida de un sujeto.

Asimismo se inicia una indagación sobre los saberes vinculados a la organización que estos actores poseen, puestos en práctica en las instancias de gestión de las que son parte; esto porque se sigue aquí una línea de estudios sobre saberes socialmente productivos, saberes a los que se define como de integración, de la política y la lucha; son aquellos que permiten, entre otras cuestiones, organizar colectivos y analizar coyunturas locales en términos políticos (Rodriguez, 2011).

El artículo difunde resultados de una investigación mayor de tipo cualitativa, en la que se ha optado por utilizar las técnicas de observación participante y entrevistas semi-estructuradas. El plan de investigación prevé plantear diálogos reflexivos con los cinco integrantes y observar su participación en las diversas actividades del PPI. En 
esta primera fase se ha entrevistado a dos de ellos, valorando que ambos tienen en común experiencias de migración y viajes, que desarrollan su actividad laboral en organismos estatales y poseen antecedentes de participación en instituciones religiosas, organizaciones sindicales y/o étnicas; así como su condición de indígenas urbanos.

El artículo contiene una breve caracterización del PPI y del escenario étnico político en el que se desarrolla, posteriormente se describen espacios y experiencias de participación surgidas en el marco del programa, se plantean lecturas iniciales de sentidos dados a estas experiencias por los sujetos; se analizan las trayectorias y saberes para la participación. Los tópicos planteados permiten presentar al final, reflexiones acerca del doble lugar de quien escribe este artículo en tanto investigadora y coordinadora del PPI, así como sobre las posibilidades de diálogos interculturales.

\section{El escenario étnico-político provincial}

Como ya se ha mencionado, el PPI se desarrolla en la Universidad Nacional del Nordeste (en adelante UNNE), institución regional ubicada en Chaco y Corrientes. La primera provincia cuenta con un importante número de población qom, wichí y moqoit. Según datos del último Censo Nacional, en Chaco 41.304 personas se reconocen como indígenas o descendientes de Pueblos Indígenas, número que constituye el 3,91 $\%$ de su población totaliii. De aquellos 41.304 , el 74,5\% pertenece al Pueblo Qom, el $11,2 \%$ al Wichí, y el 9, 4\% al Moqoit. El restante 4,9\% a otros pueblos indígenas ${ }^{\mathrm{iv}}$.

Durante las últimas décadas en América Latina, y en Argentina particularmente, se han logrado avances en derechos indígenas que alcanzan expresión en la legislación y resultan de luchas y procesos de emergencia étnica (Guarino, 2010). A esta caracterización general es necesario incorporar un análisis de escala provincial, dado que sostenemos junto con Gómez y Hadad (2009) que las estrategias desplegadas en el marco de los procesos de lucha indígena están condicionadas por procesos históricos, territorialmente específicos.

En Chaco, hacia finales del Siglo XIX y las dos primeras décadas del Siglo XX, se desarrollaron procesos complejos y en estrecha relación: el de incorporación de las tierras a la economía agro-exportadora - a través de la producción forestal, azucarera y algodonera - y el de conformación de la estructura social de este territorio (Rosso, 2011: 35). Los grupos indígenas que lo habitaban fueron despojados de sus territorios a través de campañas militares y posteriormente disciplinados para su incorporación como mano de obra temporaria de los establecimientos agrícolas, ocupando el lugar más bajo de la estratificada y étnicamente diferenciada población de la sociedad regional (Beck: 1994; Bartolomé, 2004).

Desde 1920 el sistema productivo algodonero fue el que más demanda de trabajo temporario indígena generó. Posteriormente, y fruto de políticas integracionistas, algunos grupos se convirtieron en colonos dedicados a la agricultura comercial o de subsistencia (Bartolomé, Op.cit.). La crisis algodonera iniciada en 1990 los convirtió en excluidos de la estructura social, pauperizando sus condiciones de vida y acelerando migraciones a ciudades del Chaco y Santa Fe, así como al Conurbano Bonaerense. 
Paralelamente el retorno de la democracia en la década de 1980 habilitó intensas movilizaciones para expresar demandas. Uno de los mayores logros fue la aprobación de la Ley Provincial de las comunidades indígenas en 1987, posterior a la Ley Nacional Indígena de 1985. Gómez y Hadad, (Op.cit.:12-13) destacan de la primera normativa provincial la definición de comunidad indígena y de las condiciones para su reconocimiento como sujeto de derecho a través de la tramitación de una personería jurídica, así como la creación del Instituto del Aborigen Chaqueño (en adelante, IDACH) ente autárquico gestionado por indígenas, y que significó su participación directa en la gestión de sus propios asuntos.

Tales avances legales no tuvieron como consecuencia directa el mejoramiento de las condiciones de vida de la población indígena. Hacia 2006 se produjeron una serie de movilizaciones en las que se difundió la situación crítica padecida, reivindicando el acceso a la tierra entre otras demandas urgentes ${ }^{v}$ ( Ibid.). El conflicto fue llevado hasta instancias de la Corte Suprema de Justicia de la Nación, la que obligó a los Estados nacional y provincial a garantizar a los indígenas el cumplimiento de sus derechos ${ }^{\mathrm{vi}}$. La aplicación de esa orden judicial reconfiguró las relaciones entre indígenas y Estado, dado que establecía el deber de mayor presencia indígena en instancias decisorias y en organismos estatales de salud, justicia, vivienda y educación (Rosso, Artieda y Luján, 2015).

En el ámbito educativo provincial las demandas por reconocimiento étnico y respeto a la diversidad cultural se expresan significativamente, desarrollándose procesos participativos dentro del sistema educativo con una historia de casi tres décadas. Docentes y organizaciones indígenas intervienen en la gestación y organización de instituciones de educación bilingüe e intercultural (Hecht, 2014; Artieda, Liva y Almirón, 2012). Resulta una expresión de tales avances la aprobación de la Ley de Educación Pública de Gestión Comunitaria Bilingüe e Intercultural Indígena (No. 7446/2014); en la que se establece, entre otras cuestiones sustanciales, que Consejos Comunitarios Indígenas tienen derecho a gestionar, organizar y conducir el proyecto educativo de su comunidad (artículo 9, inciso a).

Los procesos expuestos sucintamente configuran un escenario de mayor visibilidad, militancia y conciencia entre la población indígena de la provincia no exento de prácticas de cooptación y clientelismo. Procesos que impactan de manera particular en las relaciones que los indígenas aquí estudiados mantendrán con la universidad, así como en los espacios de participación disputados y/o habilitados en esta institución.

\section{Participación indígena en un programa universitario}

EI PPI se implementa desde junio de 2011 buscando contribuir con políticas y acciones a la concreción de derechos indígenas. Comprende un conjunto de ejes que responden a una perspectiva integral de lo que la Universidad está en condiciones de llevar a cabo desde las funciones de docencia, investigación y extensión ${ }^{\text {vii }}$.El principal eje de acción consiste en otorgar becas y desarrollar mecanismos de apoyo al ingreso, permanencia y egreso de jóvenes indígenas a las carreras de grado. 
La resolución de creación del programa sostiene como parte de sus fundamentos a la legislación internacional, nacional y provincial en las que se definen derechos de los pueblos indígenas que habitan el territorio viii. De este conjunto de normas se subraya el artículo $7^{\circ}$ del Convenio № 169 de la OIT, en Resolución No.733/10 (C.S.), que establece la consulta y participación en los programas y proyectos que los afecten. Asimismo se reconoce la importancia de generar mecanismos de participación a fin de garantizar la adecuada comprensión de sus necesidades y expectativas, el desarrollo de procesos de interculturalidad y la puesta en valor de la diversidad y el diálogo entre culturas. Se reconoce la complejidad y conflictividad propia de todo proyecto intercultural que supone procesos de aprendizaje e intercambio en un contexto de posiciones, historias e intereses diversos y contrapuestos (Resol. No.733/10 C.S.).

El proyecto homónimo es la estrategia de una política institucional, aprobada por el consejo superior, órgano de gobierno máximo de la Universidad. En un trabajo anterior ensayamos una narración de su breve historia, señalando que en la decisión del Rector que motorizó su creación gravitaron elementos de la coyuntura provincial. Nos referimos a las movilizaciones referidas de $2006^{\text {ix }}$. Otro elemento de la coyuntura que creemos influyó en la decisión política fue el de experiencias previas con las que contaba el mismo en extensión e investigación, además de haber sido Rector en anteriores oportunidades y Decano de Facultad ${ }^{\mathrm{x}}$. Estos antecedentes le habrían permitido acceder a información sobre otras Universidades que implementan proyectos de inclusión de jóvenes indígenas - la Universidad Nacional de Salta, por ejemplo - (Rosso, Artieda y Luján, 2012). Lo expuesto puede explicar, en parte, la decisión de diseñar e implementar el Programa.

Otro aspecto destacado fue que no se dio una discusión antes de la aprobación del Programa, entre los claustros con representación en dicho consejo. El proyecto fue redactado a pedido del Rector - por una docente e investigadora con experiencia y formación en educación indígena, y el asesoramiento de un wichí- y presentado al consejo. De manera que se trató de una definición política desde la autoridad máxima del gobierno de esta universidad sin construcción de consenso entre los actores que la integran, pero tampoco con voces de disenso. La gestación del PPI tampoco respondió directamente a demandas de indígenas y sus organizaciones por educación universitaria (Rosso, Artieda y Luján, Op.cit.).

La presencia indígena se dio desde la redacción del proyecto del PPI, ya que el representante wichí aportó a su redacción. La posibilidad de participación de este actor se dio porque se desempeñaba como colaborador de un equipo de investigación interno. En el diseño contribuyó con ideas centrales tales como definir prioridades para la selección de los aspirantes a becarios, proponer acciones de apoyo a estudiantes para evitar el abandono, por mencionar algunas.

En la interacción del líder wichí con la coordinación del programa se fue gestando la idea de formar una comisión asesora compuesta por representantes de los demás Pueblos Indígenas que habitan el Chaco. La comisión actualmente se compone por cinco representantes - tres qom, un moqoit y un wichíri -, quienes fueron elegidos por sus antecedentes de lucha. El wichí los convocó, y su convocatoria fue avalada por la universidad. Él es quien señala diferencias y semejanzas entre los miembros: 
“... venimos de diferentes organizaciones pero en muchas ocasiones, siempre hemos coincidido en pelear, luchar o exigir que se cumpla lo que está dentro de las leyes... de la constitución provincial y nacional con relación a los pueblos indígenas, entonces nosotros no hacemos más que exigirles que se cumpla lo que ya está escrito."(Representante wichí, en adelante r.w.)

A partir de la creación y funcionamiento del programa se fueron dando y disputando espacios de participación. La comisión tiene alcances consultivos y no decisorios, sin embargo, tal como se señala en un trabajo anterior, las sugerencias y opiniones acordadas en reuniones con esta comisión son consideradas en primer orden en momentos en los que la coordinación del PPI toma decisiones. En esos ámbitos, la voz de los indígenas resulta respetada y suele tener preeminencia sobre otras (Artieda y Rosso, 2013).

Respecto a su posicionamiento en la institución, la condición de asesora no resulta disonante con las características del gobierno de una universidad convencional, en la que sólo los consejos directivos de facultades y el consejo superior deciden; se trata de órganos integrados por representantes de los claustros de la institución. Los indígenas de la comisión referida no pertenecen a ningún claustro, aunque resulta válido señalar que desde 2014 esta comisión ha adquirido cierto grado de institucionalidad a través de una resolución del consejo superior en la que se aprueba su existencia y se determinan sus funciones (Resol No.1361/14) (ii $^{\text {. En la }}$ práctica realizan este trabajo de manera voluntaria no remunerada ${ }^{\text {xiii }}$, asesoran a la universidad cuando debe responder a demandas o pedidos de otros grupos indígenas; ofician de traductores, difunden información sobre el PPI y realizan acompañamiento de los estudiantes.

¿Cuáles son las tareas que la comisión debe cumplir al decir de sus miembros? En los dos entrevistados no existe un discurso homogéneo. Uno de los referentes circunscribe la tarea a lo tutorial, como la de adultos que realizan acompañamiento a los estudiantes;" ... monitorear a los chicos, buscar la forma de cómo motivarlos; porque cada uno de ellos vive una historia diferente... conversar... acercarse mejor." (Representante qom, en adelante r.q.)

El segundo propone pasos para avanzar hacia funciones decisorias "... en la medida que vaya pasando el tiempo... esta comisión tendría más acción y participación en las decisiones para ir mejorando todo lo que tenga que ver con este (sic.) programa." (r.w.)

A la vez, percibe a la comisión como un espacio de tensiones futuras, de disputa por mayor poder y participación hacia adentro de la universidad, abandonando el ropaje de las políticas tutelares.

"También... va haber más exigencia de los mismos estudiantes, de la misma universidad;... esta debe ser una acción compartida, pensada desde la multiculturalidad $^{x i v}$, la participación activa... Y lo que pasa hasta ahora es que vamos bien, ... nos damos cuenta que no solamente hay que llamar a los miembros... cuando hay un problema, sino pensarlo juntos para prever...

... incluso en el futuro... que nos den la oportunidad de elegir quien va coordinar cada año. Para que no sea siempre la gente que representa a la Universidad; romper un 
poquito el esquema tradicional de que siempre tienen que ser los blancos $y$, sobre todo formados, para que digan que deben hacer los indígenas. "(r.w.)

Finalmente este representante visualiza a la comisión como una instancia que genera aprendizajes interculturales: “... estamos muy claros que hay ignorancia en ambos lados..., esa ignorancia se va a combatir en la medida que se deje expresar y para que se hagan las cosas de acuerdo a la visión de los indígenas". (r. w.)

En una universidad convencional donde las instancias decisorias se reservan a los miembros de sus claustros ¿una comisión asesora conformada por indígenas resulta un intersticio, o es una instancia precaria para la participación? Los entrevistados la conciben desde la primera opción, como un "hueco" por el que ingresar y comenzar a ocupar espacios en una institución que históricamente los excluyó, sin dejar de reconocer la escasa institucionalidad alcanzada.

\section{De sujetos y saberes}

“... son los que saben, pero también agentes de cambio, son militantes."

Los miembros de la comisión asesora entrevistados tienen en común la migración y los viajes, desarrollan su actividad laboral en organismos estatales junto a las experiencias de participación en instituciones religiosas, organizaciones sindicales y/o étnicas; y la condición de indígenas urbanos. Tales circunstancias son consideradas aquí aspectos centrales de sus trayectorias, antes que sus niveles de escolarización en los casos estudiados esos niveles son dispares ${ }^{\mathrm{xi}}$-.

Focalizamos el análisis en las experiencias participativas, en las que creemos se producen procesos formativos. Ferry (2008) sostiene que formarse significa adquirir una cierta forma, y en tanto tal, formarse excede a la enseñanza y el aprendizaje escolares. Formarse consiste en encontrar formas para cumplir con ciertas tareas, para ejercer un oficio, una profesión, un trabajo, por ejemplo; “... uno se forma solo por mediación. Las mediaciones son variadas, diversas. Los formadores son mediadores humanos, lo son también las lecturas, las circunstancias, los accidentes de la vida, la relación con los otros." (Op.cit:54).

Se trata entonces del proceso por el cual estos actores se van convirtiendo en "militantes de la causa indígena"xvii; las circunstancias que generan formación creemos están conformadas por viajes, espacios de los que deciden ser parte, así como decisiones tomadas en determinados momentos de la vida, y que generan al decir de Alonso y Díaz (2002:30) procesos pedagógicos a partir de quiebres de rutinas. Quiebre como el narrado por uno de ellos, quien decidió migrar de Chaco a Buenos Aires para formarse en Teología como miembro de la iglesia presbiteriana.

"Mis padres no querían saber nada de que me alejara de ellos"xviii. Luego agrega, “... las decisiones que uno va tomando te van llevando a distintos lugares y uno no sabe hasta dónde puede llegar... si yo hubiese estado acá,... hubiese estado con mis amigos, hubiera hecho "changas"xix, hubiera tenido una familia, un montón de hijos pero no hubiera (sic.) tenido la experiencia que tuve..." (r.q.)

Él cambió lo que estaba prefigurado como su destino, eligió entre las oportunidades que se le presentaban en aquel momento a jóvenes qom siendo parte 
de una iglesia protestante, luego abandonó ese proyecto y comenzó su militancia étnica y sindical; finalmente volvió al Chaco.

¿Qué saberes consideran estos sujetos adquieren en dicho proceso formativo? Y ¿cuáles emergen en su desempeño como miembros de la comisión asesora del PPI? Partimos de asumir que en las organizaciones sociales se produce la circulación de un tipo de saberes a los que llamamos, junto con Rodríguez (2011), saberes socialmente productivos (en adelante SSP) ${ }^{x}$. Son aquellos saberes:

"(que)... no sólo se vinculan al trabajo sino también a otro tipo, (como los) de "integración", "ambientales", de "la política y la lucha" "xxi... saberes que permiten organizar colectivos, adaptarse a situaciones cambiantes, incorporar a los recién llegados, analizar coyunturas locales en términos políticos, ..." (Op.cit.58)

El colaborador wichí ha viajado por casi todo el continente americano. Desde esa experiencia se permite plantear algunas generalizaciones,

“... (He podido) constatar la situación de injusticia desde el Estado para con los Pueblos Indígenas como una constante."

"... (Observar) una amplitud mental (de los no indígenas) en cuanto a la comprensión de las culturas indígenas, lo que lleva a aceptar darles un trato más justo." (r.w.)

En relación con su participación en la comisión asesora del PPI, su experiencia en diversas organizaciones indígenas le da la posibilidad de conocer que "...existen consejos, espacios donde discutir,... más abiertos, no solo de asesoramiento sino también de decisión."(r.w.)

El representante qom considera que la formación adquirida por ser parte de una iglesia y por la militancia gremial, le han permitido manejarse y relacionarse con personas de distintos estratos sociales y culturales, sin sentirse en situación de desventaja.

A la vez, ambos sostienen que su cultura los dota de saberes que a instancias de su participación en la comisión asesora resultan desconocidos y valiosos para el ámbito universitario; tales como la lengua materna, la relación que como indígenas tienen con el medio ambiente pero señalan que para incorporar esos aportes hay que cambiar la relación de poder que la academia ha planteado históricamente. Al respecto el wichí sostiene,

“... se necesita escuchar la voz y la escritura del mundo indígena para que 'ellos'xxii puedan desarrollar un discurso,... entendible para el mundo occidental; porque los que escribieron hasta ahora de los indígenas fueron los académicos que solamente concluyen, analizan pero desde su mundo..." (r.w.)

Propone aportar a relaciones de valoración y respeto, así como a una revisión de lo que se entiende como lo establecido en la institución, por ejemplo la fuerte jerarquización que caracteriza a la universidad,

“... que no se trate como en el pasado que la cuestión indígena que da para Ministerio de desarrollo social; ... entonces hay que asistirle (sic.)..., sino que hay que sumarla como algo único, valioso y que puede no solamente ayudar a los que están 
estudiando, sino incluso a la misma universidad, en la forma de como relacionarse internamente, porque de lo que yo conozco, toda la estructura siempre está con un principio más foráneo, entonces hay imposiciones, ... muy fuertes, internas;... eso es lo que me parece a mí, que se puede evitar en la medida que haya espacios donde se pueda conversar y tratar..."(r.w.)

Se trata de saberes socialmente productivos en tanto “... diversos, cambiantes, no esencializados ni ahistóricos” (Rodriguez, Op.cit.:58). El conocimiento de sus culturas y lenguas, sus trayectorias y posicionamientos políticos, resultan condiciones que les permiten impulsar y ser parte de diálogos desde identidades étnicas dinámicas.

Revisando lo dicho en el epígrafe, la pregunta que surge es ¿los miembros de la comisión asesora son líderes o ancianos sabios? La respuesta no alcanza a ser concluyente, se precisan categorías flexibles que los contengan y expliquen. Estos actores no responden a la categoría de sabios que circunscriben su accionar a la comunidad indígena, tal como los define Rappaport (2007). Son militantes de la causa indígena, lo que atribuye un rasgo político a su perfil; alcanzan dominio de la oralidad y la escritura, habilidades que les permiten traspasar los límites de la comunidad, crear redes con otros grupos - indígenas y no indígenas-, producir intercambios de saberes en lo que Pratt llama la zona de contacto“... entendida como los espacios sociales en los cuales las culturas se encuentran, se chocan y se ligan en contextos de relaciones de poder asimétricas" (Pratt en Rappaport, Op.cit.:617).

\section{Inquietudes metodológicas}

Pretendo exponer brevemente el proceso atravesado al escribir este artículo y aún antes, al elaborar los tópicos que orientarían las entrevistas, por eso la redacción pasará a ser escrita en primera persona. Consulté primero textos sobre metodología etnográfica (Guber, 2001, 2004) buscando pistas y herramientas para alcanzar definiciones que permitieran acercarme a la descripción de los sujetos entrevistados. Encontré que tenía como tarea generar situaciones que favorezcan asociaciones libres. Rápidamente advertí que sería difícil lograrlo, porque mi lugar de actora del PPI ceñiría en parte, los temas sobre los que hablarían los entrevistados. Del mismo modo, creí dificultoso obtener conceptos experienciales que permitan dar cuenta del modo en que los "informantes" conciben, viven y llenan de contenido un término o una situación (Guber, 2001). Lo más probable era que las entrevistas se transformaran en una instancia de demandas y justificaciones.

En instancias de planificación, los obstáculos que imaginaba tendría me generaron un estado de inmovilidad. Ante eso pensé proponer a los actores que más bien los invitaba a reflexionar juntos sobre algunos temas, como el discurrir del programa y su participación, sus experiencias en distintos ámbitos. En consecuencia, las entrevistas se convirtieron en un intercambio reflexivo; al fin y al cabo los tres compartimos un proyecto y trabajamos para su desarrollo.

Paralelamente me generaba incomodidad pensar a los actores entrevistados como "informantes". Un texto de Mato (2005) me habilitó a seguir incómoda. Allí el autor revisa nuestras prácticas de producción de conocimientos y propone superar las contradicciones de los intelectuales de la academia que producimos textos en los que se alaba el valor de los saberes de los intelectuales y pueblos indígenas, pero basados 
en fuentes teóricas académicas; los textos y declaraciones de los indígenas cumplen funciones de provisión de datos, mientras sus autores son tratados como informantes; solapando bajo esa denominación la relación de poder establecida (Op.cit:131-132).

Al tiempo que las dudas narradas me llevaron a cuestionar el para qué de la escritura.

La investigación y comunicación de los resultados remiten al interés del investigador y la comunidad académica, sin embargo es posible revisar los modos clásicos de relación que las universidades proponen a los indígenas, desarrollar una investigación que "... resulte igualmente productiva para el etnógrafo profesional y también para la comunidad." (Rappaport, 2011:331). Las ideas y saberes que emergieron aquí animan a hacerse tales planteos.

\section{A modo de cierre}

La experiencia aquí analizada se desarrolla en un contexto de exclusión socioeconómica, signado a la vez por una intensa movilización y organización indígena en pos al logro de mayores derechos y mejores condiciones de vida. En ese marco, los indígenas del Chaco pugnan por el cumplimiento del derecho a la educación superior y a la participación. Lo analizado en el presente artículo habilita a valorar la presencia de sus representantes en la gestión de un programa que busca la inclusión a la universidad porque permite conocer sus opiniones y posiciones. En este proceso los indígenas participantes señalan la necesidad de superar prácticas de tutelaje y demandan generar políticas universitarias pensadas e implementadas en conjunto.

Los miembros de la comisión asesora poseen antecedentes valiosos para la participación ${ }^{x \times i i i}$, en instancias de gestión ponen en acto saberes no académicos, saberes para la lucha y la organización. Son quienes se mueven en zonas de contacto en las que se relacionan con otros indígenas y con no indígenas. La comisión resulta una de esas zonas, allí perciben los espacios como posibilidades, así como las tensiones y los límites de la participación en una universidad convencional.

Finalmente, sobre el proceso de investigación cuyos resultados preliminares se presentan aquí, se señala la incomodidad de la relación asimétrica entre "informante" e "investigador" percibida por la autora. Circunstancia que insta a pensar otras formas de investigar, a avanzar en propuestas de colaboración intercultural donde comunidades indígenas e investigadores se beneficien con los estudios realizados. En la misma línea se observa que los resultados aquí presentados pueden ser usados para iniciar un proceso de reflexión y análisis crítico desde ambos grupos.

\section{Bibliografía}

Alonso, G. y Díaz, R. (2002) Hacia una pedagogía de las experiencias de las mujeres. Buenos Aires, Miño y Dávila Editores.

Artieda, T. y Rosso, L. (2013) "Participación indígena en un programa institucional universitario: modos, tensiones y alcances", en VIII Jornadas de Investigación en Educación. "Educación: derechos, políticas y subjetividades". Facultad de Filosofía y Humanidades, Universidad Nacional de Córdoba; Octubre de 2013. Publicada en CD, 11 págs.

Artieda, T., Liva, Y. y Almirón, S. (2012) "La participación de docentes y organizaciones indígenas en los procesos de definición de las escuelas 
públicas de gestión social indígena en la provincia del Chaco, Argentina", en IX Congreso Internacional de la Red Estrado, Red Latinoamericana de Estudios sobre Trabajo Docente, Universidad de Santiago de Chile, Chile, 18 al 20 de julio de 2012, mimeo.

Bartolomé, M. (2004) "Los pobladores del "desierto", en Amérique Latine Histoire et Mémoire. Número 10. Identités: positionnements des groupes indiens en Amériquelatine.http://alhim.revues.org/document103.html [Consulta el oct.2015].

Beck, H. (1994) "Relaciones entre blancos e indios en los territorios nacionales del Chaco y Formosa (1885-1950)". Cuadernos de Geohistoria Regional, N²9, Chaco, Argentina, IIGHI, Conicet, Fundanord.

Ferry, G. (2008) Pedagogía de la Formación. Buenos Aires: Ediciones Novedades

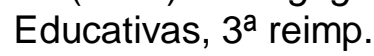

Gómez, C. y Hadad, M. G. (2009) "Manifestación de la emergencia indígena en Argentina", en XXVII Congreso de la Asociación Latinoamericana de Sociología, Asociación Latinoamericana de Sociología, Buenos Aires, mimeo.

Guarino, G. (2010) "Estrategias identitarias para la resistencia étnica en las organizaciones indígenas Qom del Chaco" en Mad, núm. 22, <http://www.revistamad.uchile.cl/index.php/RMAD/issue/view/1300> [Consulta: julio 2015].

Guber, R. (2001) La etnografía, método, campo y reflexividad. Bogotá: Grupo Editorial Norma.

-------- (2004) El salvaje metropolitano. Reconstrucción del conocimiento social en el trabajo de campo. Buenos Aires: Paidós.

Guimenez, S. (2005) Políticas sociales y los dilemas de la participación. En Laboratorio, Año 6, núm. 16, Buenos Aires, Año 6, 13-18.

Hecht, A. C. (2014) "Demandas pendientes y logros educativos. Un balance de la EIB en el Chaco entre 1987 y 2014", en XI Congreso Argentino de Antropología Social, Universidad Nacional de Rosario, 23 al 26 de julio de 2014. Disponible en línea: <http://www.11caas.org/confcientifica/comunicacionesActasEvento.php> [Consulta: oct.2015].

Mato, D. (2005) "Interculturalidad, producción de conocimientos y prácticas socioeducativas". Revista de Comunicação, Cultura e Política. Vol.6, n.11 <http://www.dhl.hegoa.ehu.es/ficheros/0000/0523/Intercult...pdf> [Consulta: nov.2015].

Mato, D. (2014) "Educación Superior y Pueblos Indígenas. Contextos, experiencias y desafíos". En Novedades Educativas, núm. 284, agosto, 2014, 11-16.

Rahnema, M. (1996) Participación, en: Sachs, W. (editor) Diccionario del desarrollo. Una guía del conocimiento como poder. Perú, PRATEC, 125-141.

Rappaport, J. (2007) "Intelectuales públicos indígenas en América Latina: una aproximación comparativa", en Revista Iberoamericana. Vol LXXIII, No.220, 615-630.

Rappaport, J. (2011) Más allá de la observación participante: la etnografía colaborativa como innovación teórica, en: A.A. V.V. Conocimientos y prácticas políticas: reflexiones desde nuestras prácticas de conocimiento situado. Tomo I. Chiapas, Ciudad de México, Ciudad de Guatemala y Lima. CIESAS, UNICACH, PDTG-UNMSM, 327-369.

Rodríguez, L. (2011) "Saberes, saberes socialmente productivos y educación de adultos", En Revista Decisio. Saberes para la acción en educación de adultos. México, CREFAL, No.30.pp. 55-60. 
Rosso, L. (2011) Educación estatal para indígenas del Chaco Argentino: tensiones históricas, en: Paladino, M. y García, S. M. (coord.) La Escolarización en los Pueblos Indígenas Americanos. Impactos y desafíos". Ecuador, Abya Yala.3561.

Rosso, L., Artieda, T.y Luján, A. (2015) Universidad y Pueblos Indígenas del Chaco. Análisis de una política de inclusión, participación e interculturalidad, en D. Mato "Educación Superior y Pueblos Indígenas en América Latina. Experiencias, interpelaciones y desafíos". Sáenz Peña, Buenos Aires, EDUNTREF, 15 págs., en prensa.

\section{Entrevistas realizadas}

Carlos Barreto, referente del Pueblo Qom en Comisión Asesora del PPI UNNE. Audencio Zamora, referente del Pueblo Wichí en Comisión Asesora del PPI UNNE.

\section{Otras fuentes}

\section{Diario}

JudicialEn:http://www.diariojudicial.com/contenidos/2007/09/19/noticia_0005.html Fecha de consulta: 3/7/2014.

Universidad Nacional del Nordeste. Resolución No.733/10 C.S., Corrientes, Argentina.

\section{Notas:}

\footnotetext{
${ }^{i}$ Siguiendo a Mato (2014) se define aquí como universidades convencionales "a aquellas [...] que no han sido explícitamente creadas y diseñadas para responder a las necesidades, demandas y propuestas de comunidades pueblos indígenas o afro-descendientes" (Mato, Op.cit:41).

ii Solo uno de ellos pertenece al culto Baha'i.

iii En: http://www.censo2010.indec.gov.ar/CuadrosDefinitivos/P1-P_Chaco.pdf

${ }^{i v}$ En Corrientes se han iniciado procesos de emergencia indígena por parte de guaraníes. El Censo 2010 arroja un total de 5.000 personas reconocidas como indígenas en esta provincia, datos que son presentados sin diferenciar a que pueblo pertenecen. En particular, se ha producido el autoreconocimiento de una comunidad del pueblo guaraní en Yahaveré (Departamento de Concepción) compuesta por 17 familias, comunidad que en 2013 adquirió personería jurídica a través del Instituto Nacional de Asuntos Indígenas.

vEntre abril y agosto de 2006 en el Chaco se produjeron importantes protestas de la población indígena y sus organizaciones ante al gobierno provincial. Las movilizaciones organizadas para expresarse tuvieron origen en el Municipio de Villa Río Bermejito (localidad situada a $350 \mathrm{kms}$. de Resistencia, la capital provincial), allí los qom fueron afectados por las inundaciones y denunciaban al intendente de aquel municipio por actos de discriminación en la entrega de recursos para paliar tal situación. Estas demandas adquirieron carácter provincial debido a que otras organizaciones adhirieron junto al IDACH y elevaron al gobierno un petitorio de nueve puntos que incluía peticiones sobre salud, educación y territorio, paralelamente se difundían denuncias sobre a entregas irregulares de tierras fiscales. De las movilizaciones se pasó en junio de aquel año a un acampe frente a la casa de gobierno provincial, y luego doce dirigentes indígenas iniciaron una huelga de hambre. Tales manifestaciones cesaron el 22 de agosto de ese año cuando las organizaciones indígenas y el IDACH firmaron un acuerdo con el gobierno provincial. Indymedia y $\quad$ Fuente: Diario Página 12http://argentina.indymedia.org/news/2006/08/435895.php;http://www.pagina12.com.ar/diario/ ultimas/20-67646-2006-05-31.html
} 
vi

Fuente:

Diario

Judicial

En:

http://www.diariojudicial.com/contenidos/2007/09/19/noticia_0005.html

Fecha de consulta: 3/7/2014.

viiEI PPI se propone las siguientes líneas de acción: I. Inclusión de pueblos indígenas en las carreras de grado de la UNNE. II. Diseño de propuestas de formación y capacitación en Pedagogía para docentes indígenas y no indígenas de escuelas de nivel inicial, primario y secundario de la provincia del Chaco. III. Actualización y capacitación de docentes, investigadores y estudiantes de la UNNE, a través de espacios de estudio y reflexión sobre Culturas Indígenas del Chaco y Educación Superior e Intercultural. IV. Diseño y ejecución de Proyectos de Extensión en función de demandas de las comunidades y organizaciones de los pueblos indígenas de la región. V. Promoción y consolidación de equipos de investigación intra e interinstitucionales sobre Pueblos Indígenas (Resol. No. 733/10 C.S.).

viii Alude a lo prescripto por la Constitución Nacional (art. 75, inciso 17), el Convenio 169 de la OIT sobre Pueblos Indígenas y Tribales (1989, adoptado por la República Argentina por Ley № 24.071), la Declaración Universal de Derechos Indígenas de la UNESCO, la Ley Nacional 23.302 de Política Indígena y apoyo a las comunidades aborígenes, La Ley de Educación Nacional 26.206, la Constitución de la provincia del Chaco (art. 37), la Ley 3.258 del Chaco (1987) de las Comunidades Indígenas, la Ley 6.604 (2010) de oficialización de las Lenguas Indígenas en el Chaco (Resol. No.733/10 C.S.)

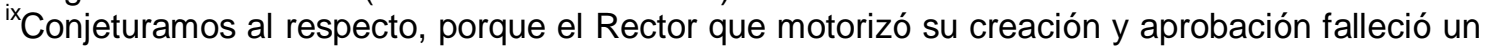
mes después.

${ }^{x}$ Señalamos también su trayectoria como funcionario del campo universitario nacional y del Estado provincial de Corrientes.

${ }^{x i}$ EI número de representantes por Pueblo trata de representar a la proporción poblacional de cada uno en el Chaco, según Censo Nacional de Población, Hogares y Viviendas. 2010, INDEC.

xii La Resolución citada establece que son funciones de la Comisión Asesora "Analizar las presentaciones de los aspirantes a becarios y recomendar la adjudicación de las becas integrales. Plantear criterios para el examen de las problemáticas presentadas por los alumnos y las alumnas surgidas en el cursado de sus carreras, y participa como "tutores culturales" transmitiendo el sentido que para las comunidades tiene el ingreso de los jóvenes a la Universidad, y acompañando a los estudiantes en la organización de actividades de creación y recreación cultural. Asesorar y ser parte de las actividades de difusión del Programa a través de la grabación de spots para medios de comunicación en las tres lenguas indígenas, definiendo contenidos y sugiriendo mejores formas de transmitir la información para promover el acceso de nuevos estudiantes de las comunidades a la Universidad."

xiii Existe un proyecto presentado por la UNNE a la Secretaría de Políticas Universitarias en el que se prevé un reconocimiento monetario a la labor de estos miembros; el mismo cuenta con aprobación pero a la fecha no se han transferido los fondos para su financiamiento. Lo aquí señalado resulta indicador de una cuestión más compleja, la ausencia de una política nacional e integral de inclusión de Pueblos Indígenas a la universidad. Las que existen son políticas institucionales, y por tanto adolecen de precariedad presupuestaria.

${ }^{\text {xiv }}$ Entendida como la presencia de varias y diferentes culturas en un territorio.

xv Frase expresada por una alumna del curso "Miradas que se cruzan: imposiciones y resistencias en la historia de las relaciones entre Pueblos Originarios, sociedad nacional y regional", dictado en el marco del PPI por indígenas, entre ellos, dos miembros de la Comisión Asesora.

xvi Uno posee primaria completa, y el otro nivel superior incompleto.

xvii La frase citada es escuchada de manera recurrente entre estos representantes y otros indígenas; puesta en contexto se usa para marcar una posición, la de reconocer una historia común de despojo de la sociedad nacional hacia los indígenas y de lucha por reclamos comunes. Posición que se enfrenta a la cooptación y sectorización ejercida por algunos partidos políticos a las organizaciones indígenas del Chaco.

xviii Representante qom.

${ }^{x i x}$ Es un término usado en la región y se usa para referirse a trabajos manuales y temporarios. 
xx Temática que presenta un amplio desarrollo y profundidad en los resultados. Son reconocidos los trabajos de Puiggrós y Gagliano (2004), Ossana (2009); y el trabajo de Rodriguez (2011) aquí citado es parte de esa línea de investigación.

xxi El encomillado pertenece a la autora citada.

xxii El entrevistado plantea que en este periodo los miembros de la universidad deben también leer a los indígenas. El sentido de este consejo remite a las publicaciones escritas que desde la década de los noventa en adelante han producido los indígenas del Chaco. La producción es diversa, comprende desde publicaciones de edición limitada escritas por maestros indígenas y para uso didáctico; a libros escritos por sabios indígenas sobre temas culturales e históricos, editados por el Estado provincial y organizaciones de la sociedad civil. El proceso de este tipo producción escrita tiene un desarrollo continuo, digno de ser investigado por especialistas interesados. Sin embargo, excede a los objetivos del presente artículo.

xxiii En párrafos anteriores señalamos que el PPI busca aportar a la concreción del derecho a la participación indígena, derecho reconocido en acuerdos internacionales y normativas nacionales; asimismo reconoce que la importancia de la participación de los indígenas a fin de garantizar la comprensión de sus necesidades y expectativas, el desarrollo de procesos de interculturalidad y la puesta en valor de la diversidad y el diálogo entre culturas (Resol. No.733/10 C.S.).

Dada la importancia que parece tener cumplir con la participación indígena se advierte que el término participación no posee un solo significado; a partir de la década de 1950 la participación aparece como una práctica estrechamente ligada a políticas públicas tendientes al desarrollo desde aquel tiempo a la actualidad su uso se ha caracterizado por ser polisémico, al tiempo que la práctica corre el riesgo de darse en formas manipuladas (Rahnema, 1996); frente a tales cuestiones ha surgido en Latinoamérica una corriente que propone el término participación popular, entendida como los esfuerzos organizados por incrementar el control sobre recursos y movimientos por parte de las clases y grupos oprimidos. El objetivo de la participación es lograr poder, un tipo especial de poder, el poder del pueblo, sus organizaciones, y en defensa de sus justos intereses (Rahnema, Op.cit:129). Por esto entendemos aquí a la participación del modo en que la define Guimenez (2005:16) como aquella que "... implica la posibilidad práctica y simbólica de intervenir en la discusión y/o en la decisión desde una posición social de libres ataduras (políticas, económicas, socioculturales), autónoma en el sentido de una práctica que pueda elevar su palabra sin miramientos, sin temores a sufrir sanciones." 